\title{
Fortalecimiento de la autonomía de jóvenes con discapacidad intelectual mediante la aplicación de las TIC
}

Camila Víquez-Alfaro* Lizbeth López-Garbanzo** Marjorie Cordero-Salas*** Pamela Alpízar-Alfaro****

*Estudiante de la Licenciatura en Educación Especial, Universidad Estatal a Distancia, Costa Rica camila.viquez@uned.cr

**Estudiante de la Licenciatura en Educación Especial, Universidad Estatal a Distancia, Costa Rica lizbeth.lopez@uned.cr

***Estudiante de la Licenciatura en Educación Especial, Universidad Estatal a Distancia, Costa Rica marjorie.cordero@uned.cr

****Estudiante de la Licenciatura en Educación Especial, Universidad Estatal a Distancia, Costa Rica pamela.alpizara@uned.cr

\begin{abstract}
Resumen
El objetivo del artículo es describir la importancia de las Tecnologías de la Información y la Comunicación (TIC) como herramientas de apoyo en los servicios de III Ciclo y Ciclo Diversificado Vocacional de Costa Rica, para el establecimiento de entornos accesibles que impulsen el desarrollo integral de la población.Se implementó la metodología de revisión bibliográfica de documentos relacionados con el tema del uso de las TIC en los procesos de enseñanza y de aprendizaje, autonomía, discapacidad y apoyos educativos; se empleó el trabajo colaborativo para establecer criterios de selección como el margen espacio temporal, terminologías específicas y área de especialización. A raíz de ello, se derivan resultados acerca de las TIC como apoyos educativos en los servicios de III Ciclo y Ciclo Diversificado Vocacional; así como su papel para el impulso de la autonomía y las consideraciones por asumir al diseñar material educativo para personas con discapacidad intelectual.
\end{abstract}

Palabras clave: Tecnologías de la información y la comunicación; tecnología educacional; acceso a la educación; igualdad de oportunidades; educación integradora; autonomización.

\section{Abstract \\ Strengthening the autonomy of young people with intellectual disabilities through the application of ICT}

The objective of the article is to describe the importance of Information and Communication Technologies (ICT) as support tools in the III Cycle and Diversified Vocational Cycle services of Costa Rica, for the establishment of accessible environments that promote the integral development of 
the population. The bibliographic review methodology of documents related to the use of ICT in the teaching and learning processes, autonomy, disability and educational supports was implemented; the collaborative work was used to establish selection criteria such as the time space margin, specific terminologies and area of specialization. As a result of this, results are derived about ICT as educational supports in the services of III Cycle and Vocational Diversified Cycle; as well as its role for the promotion of autonomy and the considerations to be assumed when designing educational material for people with intellectual disabilities.

Key words: Information and communication technologies; educational technology; access to education; equal opportunity; inclusive education; empowerment.

\section{INTRODUCCIÓN}

La sociedad se ha caracterizado por la búsqueda de mejores condiciones de vida que conllevan a la evolución, mediante la protección de los derechos como estrategia para la sana convivencia entre los ciudadanos; sin embargo, han existido poblaciones minoritarias excluidas de los ideales actuales de igualdad.

En esta misma línea, en el siglo XX durante la vigencia del modelo rehabilitador, la población con discapacidad ha tenido que enfrentar situaciones de desventaja, en las que se presentaron acciones extremistas que provocaron el rechazo y la creación de barreras participativas en cuanto a la toma de decisiones; sin embargo, desde la instauración del modelo social de la discapacidad se ha iniciado un trabajo para hacer valer los derechos, considerando que a cada individuo se le deben brindar las herramientas para la adquisición de las competencias necesarias que potencian el fortalecimiento de la autonomía, el empoderamiento y la autodeterminación (Palacios, 2008).

Debido a lo anterior, es importante identificar las formas en que las TIC pueden ser usadas como herramientas de apoyo para la facilitación de espacios de aprendizaje accesibles; indagando su aprovechamiento en el sistema educativo costarricense dirigido a jóvenes con discapacidad intelectual, población que ha presentado barreras de aprendizaje y participación ante la falta de apoyos que propicien la adquisición de nuevos conocimientos.

Asimismo, la Flórez (2015) "conceptualiza la discapacidad desde una perspectiva ecológica y multidimensional, y subraya el papel fundamental que los apoyos individualizados desempeñan en la mejora del funcionamiento humano" (p. 3); pues la discapacidad se relaciona con las barreras que el contexto pueda presentar en torno al procesamiento de la información y a las características personales en cuanto al manejo de habilidades adaptativas prácticas, conceptuales y sociales.

Actualmente, se observan avances significativos en la implementación de la tecnología en actividades cotidianas, mediante el empleo de celulares, computadoras, televisores, electrodomésticos, entre otros; "nunca antes las tecnologías habían tenido tanta presencia en la vida tanto económica, profesional, como cotidiana de la sociedad" (Alshaboul, 2012, p. 34), utilizándose según el interés de cada individuo, ya sea como medio de comunicación, movilidad, socialización, plataformas educativas o entretenimiento, siendo herramientas de acceso a un nuevo mundo de información e independencia.

Por esta razón, es fundamental que los profesionales en educación hagan uso del recurso como un apoyo didáctico, para favorecer el estímulo de habilidades adaptativas y competencias aplicables en los futuros planes de vida de la población joven con discapacidad intelectual.

Bajo esta premisa, en el artículo se pretende responder a la siguiente interrogante, ¿cómo beneficia la utilización de las TIC como apoyos didáctico, en el servicio de III Ciclo y Ciclo Diversificado Vocacional, al cual asisten jóvenes con discapacidad intelectual, para la creación de ambientes accesibles que permitan el fortalecimiento de su autonomía? 
De acuerdo con el planeamiento anterior, se analizará la eficacia de las TIC como apoyo educativo en los procesos de enseñanza y de aprendizaje, identificando aquellas que son parte del sistema de apoyos en los procesos de inclusión educativa y que favorecen la autonomía de los jóvenes con discapacidad intelectual.

Por último, se propicia bibliografía que respalda la funcionalidad del uso de las TIC como herramientas para generar ambientes educativos accesibles y de motivación que incrementen la calidad de la mediación pedagógica que impulsa el desarrollo de competencias y habilidades adaptativas en jóvenes con discapacidad intelectual.

\section{LITERATURA SOBRE EL TEMA}

A lo largo de los años, se ha reflejado un cambio en cuanto a la concepción de la discapacidad; en la antigüedad se presentó el modelo de prescindencia, en el que se observaba este aspecto desde una perspectiva religiosa, se consideraba que esta población "no contribuyen a las necesidades de la comunidad, que albergan mensajes diabólicos, que son la consecuencia del enojo de los dioses, o que - por lo desgraciadas - , sus vidas no merecen la pena ser vividas" (Palacios, 2008, p. 26), generando rechazo a razón de sus características individuales.

Para contrastar estos ideales, surgió el paradigma rehabilitador, el cual visualizó la discapacidad desde el punto de vista científico, pretendiendo normalizar a los individuos "aunque ello implique forjar a la desaparición o el ocultamiento de la diferencia que la misma discapacidad representa" (Palacios, 2008, p. 26); excluyéndolos y creando barreras de participación que por mucho tiempo perpetuaron la segregación de esta población.

Actualmente, se presenta el modelo social, el cual pretende proporcionar herramientas necesarias para asegurar el desenvolvimiento de las personas con discapacidad (PcD) en los diversos ámbitos; dirigiendo los esfuerzos a "equilibrar el acceso al ejercicio pleno de sus derechos y oportunidades en una sociedad dentro de la cual puedan desarrollar libremente y con dignidad sus propios planes y proyectos de vida" (Victoria, 2013, p. 1094), siendo así partícipes activos en el ámbito social.

En Costa Rica, la Aprobación de la Convención sobre los Derechos de las Personas con Discapacidad Ley No 8661 (2008), establece que la PcD "es sujeto de derechos y obligaciones, y no objeto de sobreprotección y/o lástima" (citado por Asamblea Legislativa de la República de Costa Rica, 2016, p. 2); por lo tanto, es obligación de la sociedad brindar las oportunidades para incorporarse independientemente en los distintos entornos.

Por otra parte, es importante mencionar que existen diversas condiciones que, en conjunto con las barreras existentes en la sociedad, generan discapacidades como la auditiva, visual, sensorial, motriz e intelectual, entre otras; sin embargo, este artículo de revisión bibliográfica se focalizará en la discapacidad intelectual.

Según la Asociación Americana de Discapacidades Intelectuales y del Desarrollo la discapacidad intelectual está "caracterizada por limitaciones significativas en el funcionamiento intelectual y en la conducta adaptativa que se manifiesta en habilidades adaptativas conceptuales, sociales y prácticas" (AAIDD, 2004; citado por Carpio, 2012, p. 15); dejando de lado las anteriores etiquetas de leve, moderado y profundo.

En el Censo nacional de población total por condición y tipo de discapacidad del 2011, se logró identificar que el total de la población que presenta una condición de discapacidad intelectual en Costa Rica es de 35416 personas; de los cuales 20966 corresponde a edades entre los 0 a los 29 años (Instituto 
Nacional de Estadística y Censos, 2011), rango de edad en el que cada joven debe asistir a los diversos sistemas educativos existentes.

Bajo esta línea, con el fin de establecer programas de estudios que potencialicen las competencias de las PCD, en Costa Rica el Ministerio de Educación Pública formuló el Plan Nacional de III Ciclo y Ciclo Diversificado Vocacional, para incrementar las posibilidades de formación e inserción sociolaboral de esta población.

El servicio citado pretende que el estudiantado pueda "desarrollar sus habilidades y destrezas para la vida y avanzar en contenidos académicos que le permitan progresar en la secuencia curricular que plantea la oferta educativa nacional, hacia el logro máximo de su autonomía y autodeterminación" (Ministerio de Educación Pública, 2014, p. 19); y así fomentar las oportunidades de inclusión.

A su vez, en Costa Rica se ha observado un progreso en cuanto al reconocimiento de esta población, mediante la aprobación de nuevas leyes que respaldan sus garantías, como lo es la Ley N. 9379 para la promoción de la autonomía personal de las personas con discapacidad (Asamblea Legislativa de la República de Costa Rica, 2016), la cual establece que todo debe tener acceso "a la asistencia personal humana y/o a los productos de apoyo que requieran para el ejercicio de este derecho, además del respeto y la promoción a la autodeterminación" (p. 3); posibilitando la independencia y el empoderamiento.

Esta ley considera que los productos de apoyo son aquellos "dispositivos, equipos, instrumentos, tecnologías, software y todas aquellas acciones y productos diseñados o disponibles en el mercado para propiciar la autonomía personal de las personas con discapacidad" (Asamblea Legislativa de la República de Costa Rica, 2016, p. 3); que faciliten el desenvolvimiento de esta población.

Al respecto, Carpio (2012) menciona que las tecnologías de apoyo han sido aplicadas desde diferentes paradigmas a lo largo del tiempo, dividiéndolas en tecnología asistiva, tecnología de la rehabilitación y TIC; en la tabla 1 se brindan los conceptos específicos de estas terminologías de manera cronológica, facilitando la comprensión de su significado.

En la tabla 1 se evidencia el avance de la terminología e implementación de la tecnología de apoyo, definidas como "cualquier producto [...], utilizado por o para personas con discapacidad destinado a

TABLA 1

Paradigmas de las tecnologías de apoyo Carpio (2012)

\begin{tabular}{|c|c|c|}
\hline \multicolumn{3}{|c|}{ Paradigmas de las tecnologías de apoyo } \\
\hline Tipo de tecnología & Años de aplicación & Características \\
\hline $\begin{array}{l}\text { La Tecnología de la } \\
\text { Rehabilitación }\end{array}$ & $\begin{array}{l}\text { Durante la década } \\
\text { de los } 1980\end{array}$ & $\begin{array}{l}\text { Utilizada para "compensar las limitaciones funcionales, para facilitar la } \\
\text { vida independiente o para favorecer que las personas mayores y las per- } \\
\text { sonas con discapacidad pudieran desarrollar todo su potencial" (Carpio, } \\
2012, \text { p. 6). }\end{array}$ \\
\hline La Tecnología Asistiva & $\begin{array}{l}\text { En 1988, se emplea } \\
\text { por primera vez esta } \\
\text { terminología y en el } \\
\text { año } 1994 \text { se incluye } \\
\text { en la legislación } \\
\text { estadounidense. }\end{array}$ & $\begin{array}{l}\text { "Configura un sector tecnológico que agrupa a todas aquellas áreas } \\
\text { científico-técnicas que pueden aportar soluciones a los problemas de la } \\
\text { accesibilidad [...] se puede estructurar en recursos (productos/equipos } \\
\text { asistivos y servicios asistivos) y estrategias (productos de apoyo y diseño } \\
\text { universal)" (Carpio, 2012, pp. 7-8). }\end{array}$ \\
\hline TIC & En la actualidad & $\begin{array}{l}\text { "Nuevo paradigma en el estímulo de las habilidades intelectuales que, a } \\
\text { lo largo del trabajo con esta población, han demostrado ser capaces de } \\
\text { desarrollar satisfactoriamente" (Carpio, 2012, p. 2). }\end{array}$ \\
\hline
\end{tabular}

Fuente: Elaboración propia basada en Carpio (2012). 
facilitar la participación, proteger, apoyar, entrenar, medir o sustituir funciones/estructuras corporales y actividades" (Abadin y Pérez, 2014, p. 6); y esenciales para impulsar competencias dirigidas a su inserción educativa y laboral.

Asimismo, se han encontrado diversos documentos que abordan el tema de las TIC como apoyos para el desarrollo integral de habilidades y competencias en jóvenes con discapacidad intelectual; a continuación se presentan en la tabla 2, organizada de forma ascendente según el año de publicación.

TABLA 2

Literatura relacionada al uso de las TIC en contextos educativos

\begin{tabular}{|c|c|c|c|c|}
\hline \multicolumn{5}{|c|}{ Literatura relacionada al uso de las TIC en contextos educativos } \\
\hline Autor & Documento & Año & Lugar & Tipo \\
\hline Ana Cristina Echeverría & $\begin{array}{l}\text { TIC para la formación inicial y permanente del } \\
\text { profesorado en educación especial: } \\
\text { Universidad de Costa Rica }\end{array}$ & 2011 & España & Tesis \\
\hline $\begin{array}{l}\text { Daniel Zappalá, Andrea Köpel y } \\
\text { Miriam Suchodolski }\end{array}$ & $\begin{array}{l}\text { Inclusión de TIC en escuelas para alumnos con } \\
\text { discapacidad intelectual }\end{array}$ & 2011 & Argentina & Libro \\
\hline María Carpio & $\begin{array}{l}\text { La tecnología asistiva como disciplina para la } \\
\text { atención pedagógica de personas con } \\
\text { discapacidad intelectual }\end{array}$ & 2012 & Costa Rica & Artículo \\
\hline Mohannad Alshaboul & $\begin{array}{l}\text { Los recursos tecnológicos y las tecnologías de la } \\
\text { información y la comunicación aplicadas a la } \\
\text { educación especial en Ammán (Jordania) }\end{array}$ & 2012 & España & Tesis \\
\hline $\begin{array}{l}\text { Agencia Europea para el Desarrollo } \\
\text { de la Educación del Alumnado con } \\
\text { Necesidades Educativas Especiales }\end{array}$ & $\begin{array}{l}\text { Tecnologías de la información y la comunicación } \\
\text { para la inclusión. Avances y oportunidades en } \\
\text { los países europeos }\end{array}$ & 2013 & $\begin{array}{c}\text { Países de la } \\
\text { Unión Europea }\end{array}$ & Libro \\
\hline Rafael Sánchez Montoya & Itinerarios inclusivos, competencias & 2013 & Costa Rica & Libro \\
\hline Darío Ríos & $\begin{array}{l}\text { La accesibilidad de las TIC en Costa Rica: Un cambio } \\
\text { disruptivo en la mente de la sociedad costarricense }\end{array}$ & 2017 & Costa Rica & Artículo \\
\hline
\end{tabular}

Fuente: Elaboración propia basada en Echeverría (2011), Zappalá, Köpel y Suchodolski (2011), Carpio (2012), Alshaboul (2012), Agencia Europea para el Desarrollo de la Educación del Alumnado con Necesidades Educativas Especiales (2013), Sánchez (2013) \& Ríos (2017).

Los documentos brindan información sustancial acerca del uso de estas herramientas en la enseñanza y el aprendizaje de la población con discapacidad, determinando a su vez que "las TIC tienen un efecto directo y cobran cada vez mayor importancia en el proceso educativo del ciudadano costarricense en su inserción a un mundo globalizado" (Cuevas \& García, 2014, p. 2); y al facilitar el acceso al mundo tecnológico.

\section{MÉTODO}

\section{Procedimiento desarrollado para la localización de las fuentes consultadas}

Diseño: con el fin de encontrar fuentes fiables, se realizó la revisión bibliográfica en documentos dedicados al uso de las TIC en los procesos de enseñanza-aprendizaje, autonomía, discapacidad y apoyos educativos. 
Estrategia de búsqueda: se llevó a cabo una búsqueda en la Web utilizando bases de datos como Dialnet, EBSCO y Google Académico; mediante la estrategia de trabajo colaborativo para la indagación de fuentes de ocho años de antigüedad, utilizando términos de búsqueda en idioma español que delimiten el tema. La bibliografía consultada se basó en el ámbito educativo de Costa Rica, Suramérica y Europa.

Criterios de inclusión y exclusión: inicialmente se recolectaron 25 documentos, de los cuales se seleccionaron dos artículos de revistas, dos leyes, dos libros, dos tesis y una normativa interna del Ministerio de Educación Pública de Costa Rica, para un total de 14 fuentes. Se establecieron una serie de restricciones que demarcan la búsqueda; descartando 11 obras que no eran inclusivas en su lenguaje o sobrepasaron el límite de años establecidos con anterioridad.

Análisis de los datos: la estructura del documento se fijó a partir de subtítulos, mencionando las TIC como apoyos educativos para PcD, consideraciones en el diseño de las mismas para jóvenes con discapacidad intelectual y su papel para el impulso de la autonomía, beneficios e implementación de éstas en el III Ciclo y Ciclo Diversificado Vocacional.

\section{RESULTADOS}

\section{TIC como apoyos educativos para PCD}

En el contexto educativo, existen medios y recursos tecnológicos que pueden ser empleados en las estrategias de mediación pedagógica, son “herramientas necesarias para la movilización, lectura, escritura, actividades de vida diaria [...] los usos son infinitos y las Tecnologías de la Información y la Comunicación no se quedan atrás en su utilidad" (Echeverría, 2011, p. 93); las cuales son oportunidades dirigidas a la población con discapacidad en entornos formativos y sociolaborales.

Del mismo modo, "la utilización de las TIC abre nuevos caminos para la comunicación y el aprendizaje, promoviendo el respeto por las diferencias y la construcción de una sociedad más justa y solidaria" (Zappalá, Köppel \& Suchodolski, 2011, p. 9); lo cual responde a ideales de equidad e inclusión derivados del modelo social de la discapacidad.

En la actualidad, existen distintas perspectivas acerca de las situaciones que influyen en la efectividad de la implementación de las TIC en procesos de enseñanza y de aprendizaje de jóvenes y adultos con discapacidad; algunos autores se centran en la individualidad de los estudiantes, en la generalidad del grupo de trabajo o en aspectos técnicos relacionados con la capacitación docente requerida para su uso.

Al respecto, autores como Zappalá et al. (2011) consideran que el "equipamiento e infraestructura no alcanza para incorporar las TIC en el aula ni para generar aprendizajes más relevantes en los estudiantes. Por ello los docentes son figuras clave en los procesos de incorporación del recurso tecnológico al trabajo pedagógico" (p. 5); los docentes brindan las herramientas necesarias para que los jóvenes sean partícipes activos en la toma de decisiones correspondientes a su proceso formativo.

Por ello, es fundamental que desde la labor docente se tome en cuenta al estudiantado para la identificación de las TIC; pues "solamente una persona con algún tipo de discapacidad puede realmente especificar con claridad a qué tipo de situaciones se enfrenta, sobre todo para alcanzar las metas" (Ríos, 2017, p. 196); el estudiantado es partícipe de los procesos de planificación y toma de decisiones.

Por otra parte, existen requerimientos esenciales por tomar en cuenta para la incorporación de las TIC en entornos educativos; como lo son la verificación de su validez, la versatilidad que proporciona en cuanto a la generalidad de los estudiantes, el acceso de los jóvenes a estas tecnologías en los hogares, 
así como la demanda de capacitación y planificación por parte de los profesores acerca de estos temas (Zappalá et al., 2011).

De igual forma, es importante señalar que "el uso de las TIC no es el objetivo final, sino un medio de apoyo a las oportunidades individuales de aprendizaje" (Agencia Europea para el Desarrollo de la Educación del Alumnado con Necesidades Educativas Especiales, 2013, p. 5); por lo que se debe reflexionar acerca de la funcionalidad de las mismas en el trabajo realizado en entornos educativos.

Desde este punto, es necesario emplear software, hardware, computadoras, tabletas y celulares, entre otros, que posibiliten que las PcD accedan a la información en igualdad de oportunidades; pues, la implementación de estas herramientas se convierte en un elemento favorecedor para empoderar al estudiantado con discapacidad. Al respecto, investigaciones como las de Nanzer \& Beltramone (2017) han comprobado que el uso de las TIC ya sea como apoyo educativo o de acceso "aumenta el nivel de autonomía del usuario" (p. 122), facilitando así su desenvolvimiento en entornos formativos.

Así mismo, autores como Torse \& Guiomar (2017) confirmaron que la utilización de programas como el GCompris facilitó "el proceso enseñanza-aprendizaje de alumnos con discapacidad intelectual posibilitando su inclusión, una vez que desarrolla sus potencialidades" (p. 255); lo cual comprueba que los softwares especializados al servicio del estudiantado, promueven las habilidades cognitivas y lo convierte en partícipe de su formación.

Por otra parte, estudios como los de Rodríguez \& Arroyo (2014) constatan que la implementación de diversas TIC como herramientas educativas, permiten no solo la inclusión del estudiantado con discapacidad en dinámicas educativas, sino también la construcción de nuevos conocimientos.

Igualmente, libros como el de Pesántez, Sánchez, Robles \& Ingavélez (2017) señalan a las TIC como apoyos en la educación de PCD, pues posibilitan el desarrollo de la autonomía mediante la accesibilidad y la inclusión; esto por medio del análisis de una recopilación de artículos relacionados con el tópico.

Sin duda, las iniciativas anteriormente mencionadas se refieren a la característica inherente de las TIC como herramientas facilitadoras en un proceso amplio que centraliza sus objetivos en el individuo; pues las diferentes acciones giran en torno al impulso de su autonomía, como eje focal para determinar los apoyos dirigidos al alcance de esta meta.

\section{Consideraciones en el diseño de TIC para jóvenes con discapacidad intelectual}

Con el fin de que las TIC sean presentadas efectivamente en la mediación pedagógica de jóvenes con discapacidad intelectual, es indispensable establecer un "diseño de materiales más simples y accesibles en sus contenidos" (Zappalá et al., 2011, p. 13), para brindar una respuesta acorde con las características de la población; por tanto, se presentará un mapa conceptual de recomendaciones en cuanto al diseño de estas tecnologías para la facilitación de aprendizajes (Figura 1).

Lo anterior, representa una concordancia entre la perspectiva de Zappalá et al. (2011), Carpio (2012), Luna (2013) y Fernández et al. (2017), ya que identifica como pensamiento en común que se deben tomar en cuenta las características del estudiantado en la selección de TIC, omitiendo la utilización de instrucciones, imágenes o características complejas en su diseño, que puedan representar una barrera de aprendizaje y participación. 


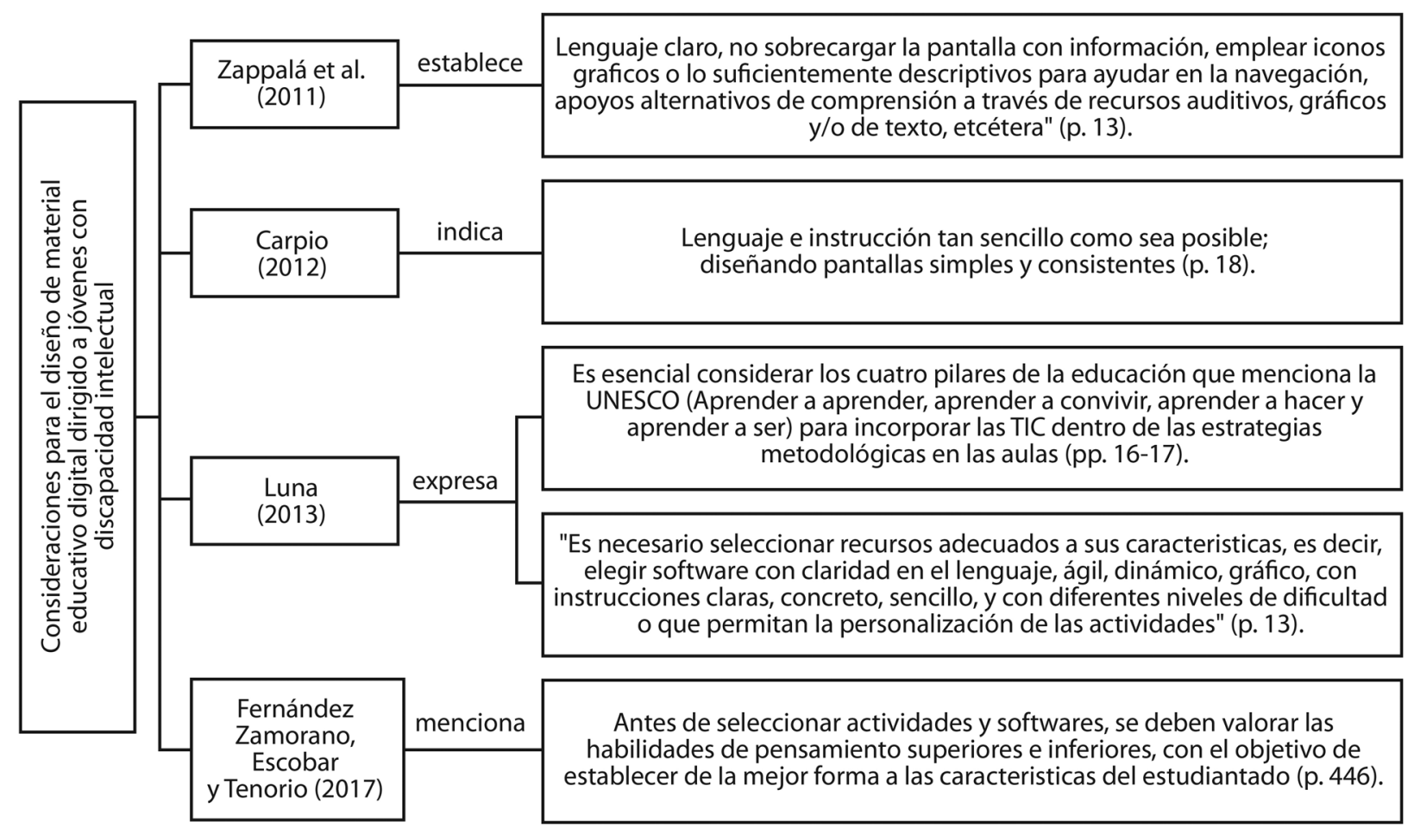

Figura 1. Mapa conceptual de recomendaciones en cuanto al diseño de estas tecnologías para la facilitación de aprendizajes. Fuente: Elaboración propia basada en Zappalá et al (2011), Carpio (2012), Luna (2013) \& Fernández et al. (2017).

\section{Uso de la computadora, Internet y software con personas con discapacidad intelectual}

El uso de herramientas tecnológicas como "la educación on-line, la tecnología del hardware y del software debe permitir a las PcD utilizar la computadora con mayor eficacia" (Carpio, 2012, p. 17); de esta forma, el usuario puede iniciar el proceso de fortalecimiento de la autonomía en el aprovechamiento de computadoras y otros dispositivos de su clase.

La perspectiva anterior es compartida por varios autores, al considerar que las computadoras son herramientas que dan respuesta a las individualidades de los estudiantes, y favorecen el proceso de enseñanza-aprendizaje y por ende aumentan así las posibilidades de efectuar planes de vida de forma exitosa; lo cual se puede evidenciar en la tabla 3.

TABLA 3

Computadoras como herramientas educativas PcD

\section{Computadoras como herramientas educativas PcD}

Zappalá et al. (2011) "Abre nuevos horizontes, nuevas formas de enseñar y oportunidades de aprender; supone la concreción de una educación inclusiva de calidad, que sea un derecho y un deber ejercido por todos (...) puede ayudar a satisfacer necesidades de comunicación y escritura" (pp. 9-10).

Alshaboul (2012) "Permite diversificar el currículo, introducir nuevas técnicas atractivas, mejora los procesos de adquisición de objetivos, etc." (p. 127).

Sánchez (2013) "Puede utilizarse de forma creativa para mejorar el desarrollo de habilidades y destrezas de los alumnos con discapacidad intelectual" (p. 212).

Fuente: Elaboración propia basada en Zappalá et al. (2011), Alshaboul (2012) \& Sánchez (2013). 
En definitiva, la tabla anterior presenta a la computadora como una herramienta enriquecedora para los estudiantes y docentes, al convertirse en un recurso significativo en la facilitación y transmisión de información; fomenta la motivación, la interacción y el servir como instrumento de apoyo para el acceso a la información y la comunicación.

Igualmente, dentro de la educación especial "las nuevas tecnologías nos proporcionan software, elementos mecánicos, electrónicos y arquitectónicos [...] que mejoran las vidas de estas personas, dándoles más independencia y permitiéndoles control de forma sencilla de determinados dispositivos" (Alshaboul, 2012, p. 99); lo cual enfatiza una vez más en el aumento de la calidad de vida de esta población.

Por lo tanto, el uso de estas herramientas tecnológicas amplía la independencia de cada individuo, al ser "un recurso que se utiliza amplia y sistemáticamente. [...] En una página web, y mediante un clic podemos partir de los aspectos más fáciles o generales, para avanzar hacia adelante o hacia atrás en las jerarquías conceptuales" (Alshaboul, 2012, p. 128); con el objetivo final de encontrar las mejores oportunidades de superación que acrecienten su bienestar personal.

Debido a lo anterior, los sujetos encargados de planificar y programar las aplicaciones de Internet y software, así como los dispositivos de hardware, deben tener como objetivo la realización de diseños agradables y de fácil acceso que propicien la utilización independiente de estas herramientas.

Desde este punto, para que los docentes logren usar efectivamente aplicaciones tecnológicas en los procesos de enseñanza y de aprendizaje, es esencial proporcionar "facilidad de instalación, adaptarse a las características funcionales de las personas, ajustarse según dificultad, tiempo y tipo de estudiante, contener sistema de evaluación, seguimiento de cada estudiante, ser motivante y conservar el interés" (Carpio, 2012, p. 18); con el fin de aprovechar al máximo las herramientas utilizadas durante de las lecciones.

En concreto, el uso del Internet y de programas multimedia adecuados para la PcD intelectual facilitará el establecimiento de actividades interactivas que sean llamativas para el estudiante, de manera que se mantengan los niveles de atención a través del dinamismo que ofrecen estos programas, como el uso de la luz, el énfasis y el movimiento (Carpio, 2012).

Por otra parte, Luna (2013) considera que las TIC ofrecen recursos multimedia que pueden favorecer los periodos de atención y concentración en PcD intelectual, por lo que ofrece algunos ejemplos de herramientas para el trabajo con esta población; como lo son los tableros de comunicación, portales especiales para el acceso a la información como el portal ZAC Browser, simuladores de realidad virtual inmersa o no inmersa y realidad aumentada, pictogramas, computadoras personales, celulares y tabletas, así como los softwares Speech Viewer, Prelingua, Modulador de voz, Globus, Vocaliza, Cuéntame, etc.

Cabe resaltar, que algunas aplicaciones que se encuentran en el Google Play pueden ser implementadas con PcD intelectual para fomentar el aprendizaje y la motivación, como lo son "Desarrollo Intelectual CAM 01", "Visual Reading - educación especial", "Smile and Learn: Juegos educativos para niños", "Visual Reading - educación especial", la gama de aplicaciones del Proyecto DANE y la de "123 Autismo" de Dokye Mobile, entre otros; con el objetivo de facilitar el proceso de enseñanza de esta población.

Bajo esta línea, es fundamental utilizar diversas estrategias metodológicas para la implementación de TIC que incentiven la participación del estudiantado, implementando el aprendizaje colaborativo, el trabajo independiente y el autoaprendizaje, realizando técnicas como la simulación de situaciones, elaboración de proyectos, exposiciones, debates, simposios, análisis y diálogo sobre un video o tema específico, entre otros (Latorre \& Seco, 2013, p. 20).

Al respecto, el Ministerio de Educación Nacional de Colombia (2011) menciona que se deben utilizar diferentes vías sensoriales, tomar en cuenta características, ritmos y estilos de aprendizaje, realizar 
planeamientos interdisciplinarios, usar la estrategia de trabajo cooperativo y ofrecer actividades, recursos y procedimientos que representen apoyos educativos para el logro de aprendizajes significativos (p. 49, citado por González \& Ramírez, 2012).

\section{Papel de las TIC para el impulso de la autonomía}

Como eje fundamental en los procesos educativos, debe guardarse una proporcionalidad con respecto a los lineamientos dispuestos en la Ley 9379 para la promoción de la autonomía de las personas con discapacidad, la cual considera que esta población tiene derecho "a construir su propio proyecto de vida, de manera independiente, controlando, afrontando, tomando y ejecutando sus propias decisiones en los ámbitos público y privado" (Asamblea Legislativa de la República de Costa Rica, 2016, p. 2); al ser fundamental promover la independencia y aprendizajes para la vida desde la formación en los sistemas educativos.

De igual forma, gracias a las TIC es posible "realizar tareas complejas de manera simplificada al detallar los procedimientos para la realización de éstas, actuando como modelos en el trabajo autónomo de cada estudiante" (Carpio, 2012, p. 16); lo cual propicia el empoderamiento y la autodeterminación.

Asimismo, se otorga un valor instrumental y mediador para el fomento de habilidades necesarias iniciadas en el campo educativo con sensibilidad a ser transferidas a la cotidianidad, pues las TIC estimulan "competencias participativas, que se relacionarían con el aprender a convivir o saber estar, y que hacen posible que el usuario y usuaria pueda participar en su entorno social" (Sánchez, 2006, p. 6); lo cual aumenta las posibilidades de tomar las decisiones relacionadas con su vida personal.

Por otra parte, según las Naciones Unidas el acceso a las TIC es otro aspecto prioritario en el "entorno físico, social, económico y cultural, de la salud, la educación, la información y las comunicaciones para que las PcD puedan gozar plenamente de todos los derechos humanos y las libertades fundamentales" (citado por Agencia Europea para el Desarrollo de la Educación del Alumnado con Necesidades Educativas Especiales, 2013, p. 11); lo que asegura el ejercicio pleno de las garantías que promueven la autonomía personal.

\section{Beneficios del uso de las TIC con jóvenes con discapacidad intelectual}

El uso de las TIC en personas con discapacidad intelectual genera beneficios durante el proceso de formación, tanto para los docentes como para los estudiantes, los cuales representan "una mejora cualitativa de los procesos de enseñanza y de aprendizaje, desarrollar capacidades y competencias, atender a la singularidad y a las necesidades individuales de cada alumno y potenciar motivaciones que den un carácter significativo a los aprendizajes" (Zappalá, et al., 2011, p. 8), y promueven una mediación pedagógica que responda a las características de cada individuo; tales aspectos positivos se presentan en la tabla 4.

Las perspectivas de estos autores establecen a las TIC como factores propicios para el diseño de entornos inclusivos, que estimulen la autonomía y la autodeterminación en jóvenes con discapacidad intelectual; generando procesos de enseñanza y de aprendizaje basados en ideales de respeto y equidad.

Asimismo, las TIC estimulan las capacidades cognitivas como la "memoria, atención, concentración, cálculo" (Carpio, 2012, p. 19); al fortalecer habilidades prácticas, sociales y conceptuales necesarias para que una PcD intelectual pueda relacionarse de forma independiente en diversos ámbitos.

Cabe mencionar que todos estos beneficios se relacionan con dos aspectos, uno de ellos es el sentimiento que provoca en los individuos un correcto empleo de las TIC y el otro al incrementar el acceso 
TABLA 4

Ventajas de la implementación de las TIC como apoyo educativo

Ventajas de la implementación de las TIC como apoyo educativo

Carpio (2012) Ofrecen "motivación hacia el aprendizaje, incremento de la autoestima, facilitación de la práctica y repetición, la interactividad y la individualización de la enseñanza" (p. 17).

Zappalá et al. (2011) Podrían representar apoyos ante barreras de aprendizaje y participación, permitiendo potenciar el fortalecimiento de competencias y el logro de los objetivos; facilitando el acceso a mundos desconocidos para quienes sufren cierta exclusión social" (p. 9).

Ríos (2017) Propician "oportunidades para todas las personas, basándose en conceptos de universalidad y bienestar social" (p. 180).

Sánchez (2013)

Los jóvenes con discapacidad intelectual "a través de los juegos y simulaciones, adquieren un compromiso con el cumplimiento de una misión, habilidad para desarrollar estrategias complicadas o la capacidad de hacer planes y adelantarse a los acontecimientos" (p. 25).

Fuente: Elaboración propia basada en Zappalá et al. (2011), Ríos (2017) \& Sánchez (2013).

universal en los diversos entornos (Carpio, 2012); creando entornos accesibles, flexibles y de motivación que propicien la autonomía y empoderamiento en los estudiantes.

Sin embargo, a pesar de las múltiples ventajas que ofrecen las TIC en la población con discapacidad, se debe tener cuidado de implementarlas "con prudencia y evitando información nociva o ilícita y toma de conciencia de la necesidad de controlar el tiempo que se dedica al entretenimiento con las TIC así como de su posible poder de adicción (uso o abuso)" (Alshaboul, 2012, p. 62); es fundamental que los docentes recuerden que el objetivo final es utilizarlas como apoyos y no como centro del aprendizaje, estableciendo al estudiante como principal actor de los procesos formativos.

\section{Implementación de TIC en III Ciclo y Ciclo Diversificado Vocacional en Costa Rica}

En Costa Rica se pretende generar ambientes inclusivos, por lo que se instauró el plan de estudios de III Ciclo y Ciclo Diversificado Vocacional, cuyo propósito es facilitar en los estudiantes "el desarrollo máximo de sus potencialidades, autonomía, independencia y formación para el desempeño de una actividad productiva/laboral o bien, la continuidad del proceso educativo en otras ofertas formativas o educativas [...] esencial en la construcción del proyecto de vida" (Ministerio de Educación Pública, 2014, p. 16), y abrir así mayores posibilidades de ser sujetos activos dentro de la sociedad.

De esta manera, con el objetivo de eliminar la exclusión educativa y disminuir el porcentaje de desempleo de personas adultas con alguna condición de discapacidad, el Ministerio de Educación Pública estableció un currículo constituido por áreas académicas y conocimientos técnicos que resulten en procesos de inserción sociolaboral exitosos.

Bajo esta línea, el plan abarca el III Ciclo (sétimo, octavo y noveno), en el que se realiza "un periodo de exploración de las destrezas y habilidades del estudiantado por medio de su participación en una variedad de experiencias y actividades relacionadas con procesos productivos/laborales" (Ministerio de Educación Pública, 2014, p. 30); y así comenzar un proceso de ubicación vocacional que responda a las características, deseos y expectativas de los estudiantes. 
Lo anterior, como proceso inicial para el ingreso al Ciclo Diversificado Vocacional conformado por décimo, undécimo y duodécimo año, estableciendo un "periodo de formación vocacional, en el que se brinda al estudiante las posibilidades de formación en un área específica, según sus habilidades y destrezas" (Ministerio de Educación Pública, 2014, pp. 30-31); con el fin de incrementar las futuras oportunidades laborales de esta población.

A su vez, el plan (Ministerio de Educación Pública, 2014, p. 28) pretende utilizar "los recursos pedagógicos (videos, tecnologías de información y comunicación, guías, juegos, libros, fichas diccionarios, periódicos, entre otros)" en los procesos de planificación, para generar ambientes accesibles, motivadores e interactivos que posibilitan la construcción de aprendizajes más significativos.

Debido a esta propuesta, se logra reconocer "que se han realizado mejores avances en las actividades que incluyen a las TIC en Costa Rica, pero aún falta gran camino por recorrer" (Ríos, 2017, p. 192); es decir, existe un vacío en cuanto al máximo aprovechamiento de los recursos, ya sea por falta de capacitación en su uso o por la inaccesibilidad a las opciones tecnológicas existentes.

Igualmente, Echeverría (2011) considera que en Costa Rica se puede acceder a las TIC por medio de recursos formales e informales, sin embargo existen dos barreras significativas en cuanto a su adquisición y aplicación; el costo económico de la tecnología y el requerimiento constante de capacitación para el aprovechamiento máximo de las TIC.

Aunado a esto, la Universidad de Costa Rica indica que las PcD se encuentran en desventaja en cuanto al acceso a las TIC; esto debido a que la población "está altamente vinculada a la pobreza y existe una alta correlación entre disponibilidad de medios económicos y acceso real a las TIC" (UCR, 2003; citado por Ríos, 2017, p. 194), lo cual representa un obstáculo para su obtención.

Es importante que Costa Rica sea reconocida como "una nación en búsqueda de igualdad de oportunidades, desarrollo personal y profesional para las personas, independientemente de sus condiciones físicas y mentales" (Ríos, 2017, p. 195); por lo que es esencial que las TIC sean empleadas como herramientas de acceso para la facilitación no solo de aprendizajes, sino como medios de apoyo para el mejoramiento de la calidad de los procesos de mediación pedagógica que propicien el desarrollo de competencias que fortalezcan la autonomía de la PcD.

\section{CONCLUSIONES}

Durante la revisión bibliográfica se logró adquirir información valiosa en cuanto a las TIC como coadyuvantes en el proceso educativo, rescatando documentos de diversos lugares y años que concuerdan en ideales relacionados a la importancia de respetar la individualidad de las PCD, al implementar las TIC como parte de sus sistemas de apoyos.

A su vez, estos documentos identificaron la importancia de las TIC como herramientas de apoyo en la mediación pedagógica de jóvenes con discapacidad intelectual, siendo elementos enriquecedores que aumentan las posibilidades de producir ambientes accesibles que impulsen su autonomía.

Los autores Alshaboul (2012), Carpio (2012), Luna (2013) y Pesántez et al. (2017), permitieron comprobar como la utilización de las TIC potencian la autonomía en PCD, por medio de contextos inclusivos que impulsen la comprensión de aprendizajes y enfoquen la planificación en el beneficio de cada estudiante.

Es fundamental reconocer que todas las personas poseen el derecho de desenvolverse de forma independiente en la sociedad, por lo que es esencial que los docentes planifiquen los medios necesarios para el fortalecimiento de competencias y habilidades en ámbitos formativos. 
En Costa Rica, se ha logrado identificar la aplicación de TIC en el III Ciclo y Ciclo Diversificado Vocacional, sin embargo los autores Echeverría (2011), Ríos (2017) y la UCR (2003; citada por Ríos, 2017) concuerdan en que la tecnología en este contexto tiene un costo económico significativo que provoca que gran parte de la población con discapacidad no pueda acceder fácilmente a ella; por tanto, es fundamental valorar esta situación con el objetivo de brindar un mayor provecho de las TIC en el sistema educativo costarricense.

Sin embargo, a partir de los resultados generados mediante la revisión bibliográfica, se puede recomendar la implementación de TIC en entornos educativos como medio para enriquecer el aprendizaje, tomando en cuenta al estudiantado y sus características en la planificación; siendo fundamental, asumir las consideraciones brindadas por Zappalá et al. (2011), Carpio (2012), Luna (2013) y Fernández et al. (2017) con respecto al diseño de TIC, para que su selección favorezca el fortalecimiento de los aprendizajes de las PcD intelectual.

A su vez, es esencial que se utilicen las estrategias metodológicas mencionadas tanto por Latorre \& Seco (2013) como las citadas por González \& Ramírez (2012), ente ellas el trabajo colaborativo y el autoaprendizaje, de la mano con páginas multimedia, software, hardware y aplicaciones didácticas, por ejemplo, las del Proyecto DANE y de "123 Autismo" de Dokye Mobile; lo anterior, con el objetivo de aumentar la motivación e independencia en la aplicación de actividades educativas que generen entornos inclusivos e impulsen el desarrollo autónomo e integral de las PcD intelectual.

Finalmente, la literatura analizada permitió encontrar una relación entre la aplicación de las TIC como factor importante para el desarrollo de entornos formativos accesibles y el impulso de habilidades adaptativas que deriven en una mayor autonomía; la cual responde a las características, deseos e intereses de los estudiantes al fomentar la calidad de los procesos pedagógicos.

\section{REFERENCIAS}

Abadin, A. \& Pérez, L. (2014). Tecnologías de apoyo, mercado y nuevos sistemas de información. Recuperado de http://www.ceapat.es/InterPresent1/groups/imserso/documents/binario/tecnologiaapoyo.pdf

Agencia Europea para el Desarrollo de la Educación del Alumnado con Necesidades Educativas Especiales (2013). Tecnologías de la información y la comunicación para la inclusión. Avances y oportunidades en los paises europeos. Recuperado de https://www.european-agency.org/sites/default/ files/ICT_for_Inclusion-ES.pdf

Alshaboul, M. (2012). Los recursos tecnológicos y las tecnologías de la información y la comunicación aplicadas a la educación especial en Ammán (Jordania) (Tesis doctoral, Universidad de Granada, España). Recuperado de http://digibug.ugr.es/handle/10481/21759

Asamblea Legislativa de la República de Costa Rica (2016). Ley N 9379, ley para la promoción de la autonomía personal de las personas con discapacidad. Recuperado de http://www.tse.go.cr/pdf/normativa/ promocionautonomiapersonal.pdf

Carpio, M. (2012). La tecnología asistiva como disciplina para la atención pedagógica de personas con discapacidad intelectual. Actualidades investigativas en educación, 12(2), 1-27 Recuperado de http:// www.redalyc.org/pdf/447/44723437018.pdf

Cuevas, F. \& García, J. (2014) Las TIC en la formación docente. Congreso Iberoamericano de Ciencia, Tecnología, Innovación y Educación, 1159. Recuperado de https://www.oei.es/historico/congreso2014/memoriactei/1159.pdf

Echeverría, A. (2011). Tics para la formación inicial y permanente del profesorado en educación especial: Universidad de Costa Rica. (Tesis de doctorado, Universidad Complutense de Madrid, España). Recuperado de http://eprints.ucm.es/12593/1/T32960.pdf 
Fernández, K., Zamorano, I., Escobar, P. \& Tenorio, M. (2017). Inclusión, discapacidad y educación: Enfoque práctico desde las Tecnologías Emergentes. Recuperado de https://www.researchgate.net/ publication/317551722

Flórez, J. (2015). Discapacidad intelectual: ¿Qué es? ¿Qué define? ¿Qué se pretende?. Recuperado de https:// www.downciclopedia.org/images/neurobiologia/Discapacidad-intelectual.pdf

González, A. \& Ramírez, J. (2012). Estrategias pedagógicas alternativas en pausas activas para la inclusión educativa de escolares con discapacidad cognitiva en Institución Villa Santana del municipio de Pereira. (Tesis de Licenciatura, Universidad Tecnológica De Pereira, Colombia). Recuperado de http://repositorio.utp.edu.co/dspace/bitstream/handle/11059/2708/37192886132G643. pdf?sequence $=1 \&$ isAllowed $=y$

Instituto Nacional de Estadística y Censos (2011). Costa Rica: Población total por condición y tipo de discapacidad, según sexo y grupos de edad. Recuperado de http://www.inec.go.cr/system/files_force/ documentos/poblacion/estadisticas/resultados/repoblaccenso2011-07.xls.xls?download=1

Latorre, M. \& Seco, C. (2013). Metodología. Estrategias y técnicas metodológicas. Recuperado de http:// www.umch.edu.pe/arch/hnomarino/metodo.pdf

Luna, M. (2013). Tecnología y discapacidad: Una mirada pedagógica. Revista Digital Universitaria, 12(14), 1-19. Recuperado de http://www.revista.unam.mx/vol.14/num12/art53/art53.pdf

Ministerio de Educación Pública (2014). Orientaciones Técnicas para la Implementación del Plan de Estudios de III Ciclo y Ciclo Diversificado Vocacional. Recuperado de https://cenarec.files.wordpress. com/2016/02/orientaciones-tc3a9cnicas-iii-ciclo-diversificado-2014.pdf

Nanzer G. \& Beltramone, D. (2017). Kit Open Source de ayudas técnicas para actividades de la vida diaria de personas con dificultad de movimiento en manos. Recuperado de https://www.researchgate.net/ publication/317551722

Palacios, A. (2008). El modelo social de discapacidad: orígenes, caracterización y plasmación en la Convención Internacional sobre los Derechos de las Personas con Discapacidad. Recuperado de https://www.cermi.es/sites/default/files/docs/colecciones/Elmodelosocialdediscapacidad.pdf

Pesántez F., Sánchez, S., Robles, V. \& Ingavélez, P. (2017) Inclusión, discapacidad y educación. Enfoque práctico desde las Tecnologías Emergentes. Recuperado de https://www.researchgate.net/ publication/317551722

Ríos, D. (2017). La accesibilidad de las TIC en Costa Rica: Un cambio disruptivo en la mente de la sociedad costarricense. Revista Latinoamericana de Derechos Humanos, 28(1). Recuperado de http://www.revistas.una.ac.cr/index.php/derechoshumanos/article/view/9660/11844

Rodríguez, M. \& Arroyo, M. (2014). Las TIC al servicio de la inclusión educativa. Digital Education Review, (25), 108-126. Recuperado de http://revistes.ub.edu/index.php/der/article/viewFile/11331/pdf

Sánchez, R. (2007). Las tecnologías en la escuela inclusiva: nuevos escenarios, nuevas oportunidades. Didáctica, innovación y multimedia, 9, 1-10. Recuperado de https://www.raco.cat/index.php/DIM/ article/view/73622/85301

Sánchez, R. (2013). Itinerarios inclusivos, competencias. San José, Costa Rica: EUNED.

Torse, I. \& Guiomar E. (2017). Utilizando o software GCompris na aprendizagem matemática com alunos com deficiência intelectual.Recuperado de https://www.researchgate.net/publication/317551722

Victoria, J. (2013). El modelo social de la discapacidad: una cuestión de derechos humanos. Boletín Mexicano de Derecho Comparado, 138, 1093- 1109. Recuperado de http://www.scielo.org.mx/pdf/ bmdc/v46n138/v46n138a8.pdf

Zappalá, D., Köppel, A. \& Suchodolski, M. (2011). Inclusión de TIC en escuelas para alumnos con discapacidad intelectual. Recuperado de http://escritorioeducacionespecial.educ.ar/datos/recursos/pdf/m-intelectuales-1-40.pdf 